\title{
Automated Real-Time Measurement of Chemotactic Cell Motility
}

BioTechniques 31:1130-1138 (November 2001)

\author{
Nacima Hadjout, Gary \\ Laevsky, David A. Knecht, \\ and Michael A. Lynes \\ University of Connecticut, \\ Storrs, CT, USA
}

\section{INTRODUCTION}

Cell movement is fundamental to many important biological processes; cells move during organogenesis, movement is essential to inflammatory immune responses, and the movement of neoplastic cells permits metastasis to secondary sites. This movement can arise from the intrinsic characteristics of the cell, or it can be initiated or enhanced by the presence of external stim uli. Stimuli can be divided into two classes, those that stimulate cell movement without a specific directional aspect (chemokinesis) and those that enhance directional cell movement according to the location of external cues (chemotaxis). The measurement of cell movement directed by chemotactic agents has been accomplished in several ways. The "small-population" assay (7) optically measures the movement of cells in an initial localized deposit of these cells in a chemotactic gradient that exists within the agarose below the cells. Variations of the Boyden chamber assay (1) are currently the most com monly used. In these assays, the cells are placed on a microporous membrane over a source of chemotactic agent. As the cells detect the higher concentrations of chemotactic agent that diffuse from the source, they migrate through the membrane to its underside. Migrating cells are usually detected on the reverse side of the membrane after staining. Responding cells are usually enumerated as an endpoint assay at a predetermined time point. An advantage of this technique is the ability to perform many simultaneous assays in parallel. A major limitation of the Boyden assay is that the gradient is very steep and dissipates rapidly (11). It is also difficult to visualize the movement of cells through the filter in this chemotactic environment. Another technique used to measure chemotaxis is to track cell movement by video microscopy in a Zigmond or Dunn chamber $(29,30)$. In these assays, the movement of cells is recorded as they respond to an aqueous gradient of chemoattractant formed between two closely spaced glass surfaces. This assay is more difficult to set up, only a small number of cells can be analyzed at a time, and the assay cannot be easily multiplexed.

The under-agarose chemotactic assay $(17,18)$ provides a different environment from that found in other assays. First, the cells move while they are surrounded by the underlying substrate and the overlying agarose. Second, the chemotactic gradient is stabilized by the agarose, allowing the gradient to be established over a larger area for a longer time. The traditional under-agarose assay measures the distance that the cells move in a specified period of time as an indication of a chemotactic response. This assay has the advantage that a single endpoint need not be evaluated because the cells gradually spread away from the starting well. The disadvantage is that in running many parallel assays, each would have to be evaluated microscopically at many time points to get an estimate of the extent of movement in each assay. We have adapted the under-agarose assay to be compatible with the automated assessment capability of the electric cell impedance sensing (ECIS) (6) system to create the ECIS/taxis system.

In the ECIS assay system, two electrodes are lithographed on the surface of 
a lexan slide and positioned within a chamber that holds aqueous media. Cells in this media can attach to the small electrode and to the surrounding surface of the slide. A one volt a.c. current passes through the culture media electrolyte, and a lock-in amplifier measures the current flow through this circuit, detecting the initial resistance of the system and any changes to the current flow that occur over time. The resistance at the small electrode predominates in this system (6). Events or agents that alter the adherence of cells to the small electrode will alter the electrical resistance in the system. For example, increasing the tightness of cell association with the electrode surface by coating the surface with extracellular matrix proteins increases the resistance of the electrical circuit (27). Lipopolysaccharide activates macrophages to spread and cover a larger amount of the target electrode and, thus, also increase the resistance measured at the target electrode (9). In contrast, toxicants that damage cells will act to reduce the resistance of the circuit (5).

In the studies described here, we have used Dictyostelium discoideumto study eukaryotic cell chemotactic movement. These cells thrive at ambient conditions and their mechanisms of motility are analogous to leukocytes (3). In moving across the substrate, these cells extend pseudopodia at their leading edge that attach to the substrate and orient the cell in the direction of travel. Dictyostelium are chemotactic to a variety of agents. For example, folic acid produced by bacteria establishes a gradient that allows vegetative Dictyostelium cells to find their bacterial prey. Cyclic AMP (cAMP), a chem otactic signal produced by Dictyostelium, is used during development to direct the aggregation of individual cells to form the multicellular organism (2). Using this system, we have shown that the ECIS/taxis modifications enable the establishment of a stable chemotactic gradient in which cell responses can be measured in real time. The ECIS/taxis technique is sufficiently sensitive to detect the arrival of a single cell at the surface of the target electrode. Moreover, the arrival time of migrating cells at the target electrode is relatively uniform and enables the identification of a wave-like behavior in the movement of these cells. The results presented here demonstrate that the ECIS/taxis system can be used to characterize chemoattractants, soluble antagonists of chemotaxis, or novel mutants affecting chemotaxis.

\section{MATERIALS AND METHODS}

\section{Cells}

D. discoidium strain $\mathrm{NC} 4 \mathrm{~A} 2$ is an axenic cell line derived from the wildtype NC4 line (15). The myosin II heavy chain mutant (HK323) was generated by homologous recombination to delete the coding portion of the gene in the NC4A2 cell line (25). Cells were maintained in HL5 media (26) in 100$\mathrm{mm}$ Petri dishes, with media changes every three days. Cells that were to be used for experimental procedures were harvested at mid-log phase. The cells were centrifuged at $200 \times g$ for $5 \mathrm{~min}$ at room temperature, resuspended in fresh media, and counted with a Z2 particle counter (Beckman Coulter, Miami, FL, USA). ECIS/taxis chambers were loaded with $10^{6}$ cells/well.

\section{Agarose Preparation}

Chemotaxis assays were adapted from the under-agarose chemotaxis method and are described in detail in the accompanying manuscript (10). Briefly, a $0.5 \%$ solution of GTG agarose (BMA, Rockland, ME, USA) was prepared in $1 \times \mathrm{SM}$ media [10 $\mathrm{g}$ Bacto-Peptone (Difco, Detroit, MI,

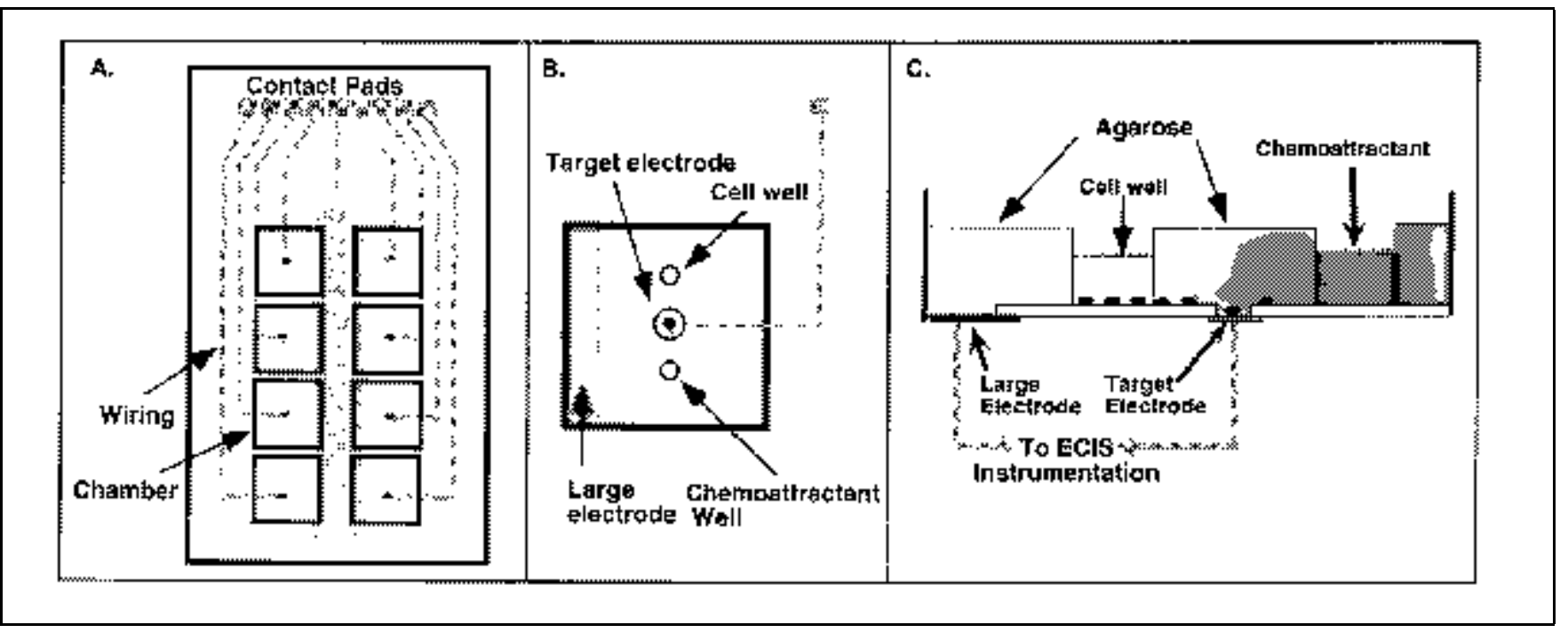

Figure 1. Schematic configuration of ECIS/taxis chambers. (A) Top view of ECIS/taxis eight-chambers array. The standard ECIS/taxis slide is composed of eight chambers that can be used to assess eight different samples simultaneously. The contact pads are connected to the ECIS/taxis instrumentation. (B) Enlarged top view of ECIS/taxis chamber. Each chamber contains a target electrode and a large common electrode. Agarose is added to the chamber, and then the cell and chemoattractant wells are cut $2 \mathrm{~mm}$ from the target electrode. (C) Side view of ECIS/taxis chamber. As the chemotactic gradient is established in the agarose overlay of the chamber, cells that sense the gradient move out of the well underneath the agarose toward the higher concentrations of chemoattractant. The migrating cells will eventually arrive at the target electrode and cause the resistance to increase. The cells are represented as filled ovals migrating across the photoresist surface toward the target electrode. 
USA), $10 \mathrm{~g}$ glucose, $1 \mathrm{~g}$ yeast extract, $1.9 \mathrm{~g} \mathrm{KH}_{2} \mathrm{PO}_{4}, 0.6 \mathrm{~g} \mathrm{~K}_{2} \mathrm{HPO}_{4}, 0.43 \mathrm{~g}$ $\mathrm{MgSO}_{4} / \mathrm{L}, \mathrm{pH}$ 6.5]. For chemokinetic assays, $2 \times \mathrm{SM}$ agarose media were premixed with an equal volume of $2 \times$ folic acid and then added to the ECIS/taxis chamber to harden.

\section{Reagents}

The chemoattractant folic acid stock (100 mM) (Research Organics, Cleveland, OH, USA) was prepared by dissolving $0.44 \mathrm{~g}$ folate in $220 \mu \mathrm{L} 10 \mathrm{M}$ $\mathrm{NaOH}$. The final volume was adjusted to $10 \mathrm{~mL}$ with distilled water. The solution was filter sterilized through a $0.2-\mu \mathrm{m}$ filter, aliquoted, and stored frozen at $-20^{\circ} \mathrm{C}$ in the dark. The folate solution was adjusted to the appropriate concentration and added to ECIS/taxis wells $1 \mathrm{~h}$ before the addition of the cells to allow for the establishment of the chemotactic gradient. Cisplatin (Sigma, St. Louis, MO, USA) (21) was dissolved in PBS ( $8 \mathrm{~g} \mathrm{NaCl}, 0.2 \mathrm{~g} \mathrm{KCl}, 0.2$ g $\mathrm{KH}_{2} \mathrm{PO}_{4}, 1.15 \mathrm{~g} \mathrm{Na}_{2} \mathrm{HPO}_{4}$ in 1000 $\mathrm{mL}$ distilled water), and the cells were incubated with three different concentrations of cisplatin in PDF $(20 \mathrm{mM}$ $\mathrm{KCl}, 5 \mathrm{mM} \mathrm{MgCl}_{2}, 20 \mathrm{mM} \mathrm{KPO}_{4}$, $0.5 \%$ dihydrostreptomycin sulfate, $\mathrm{pH}$ 6.4) for $1 \mathrm{~h}$. Following this incubation, the cells were washed three times in PDF and then resuspended in PDF before placement in the cell wells of ECIS/taxis chambers.

\section{ECIS/Taxis System}

The current ECIS electrode configuration (Applied Biophysics, Troy, NY, USA) consists of eight chambers per array (6), each with a large electrode and a small target electrode (Figure 1A). These chambers were filled with 300 $\mu \mathrm{L} 0.5 \%$ solution of melted agarose, prepared as described above. The thickness of the agarose layer is $4 \mathrm{~mm}$. After the agarose had solidified, a sharpened 14-gauge cannula (Becton Dickinson, Franklin Lakes, NJ, USA) was used to punch wells at appropriate locations in the agarose. For chemotaxis assays, wells were located $2 \mathrm{~mm}$ on either side of the target electrode, along a common axis (Figure 1B). The chambers were chilled at $4^{\circ} \mathrm{C}$ for $15 \mathrm{~min}$, and then the agarose plugs were removed by aspira-

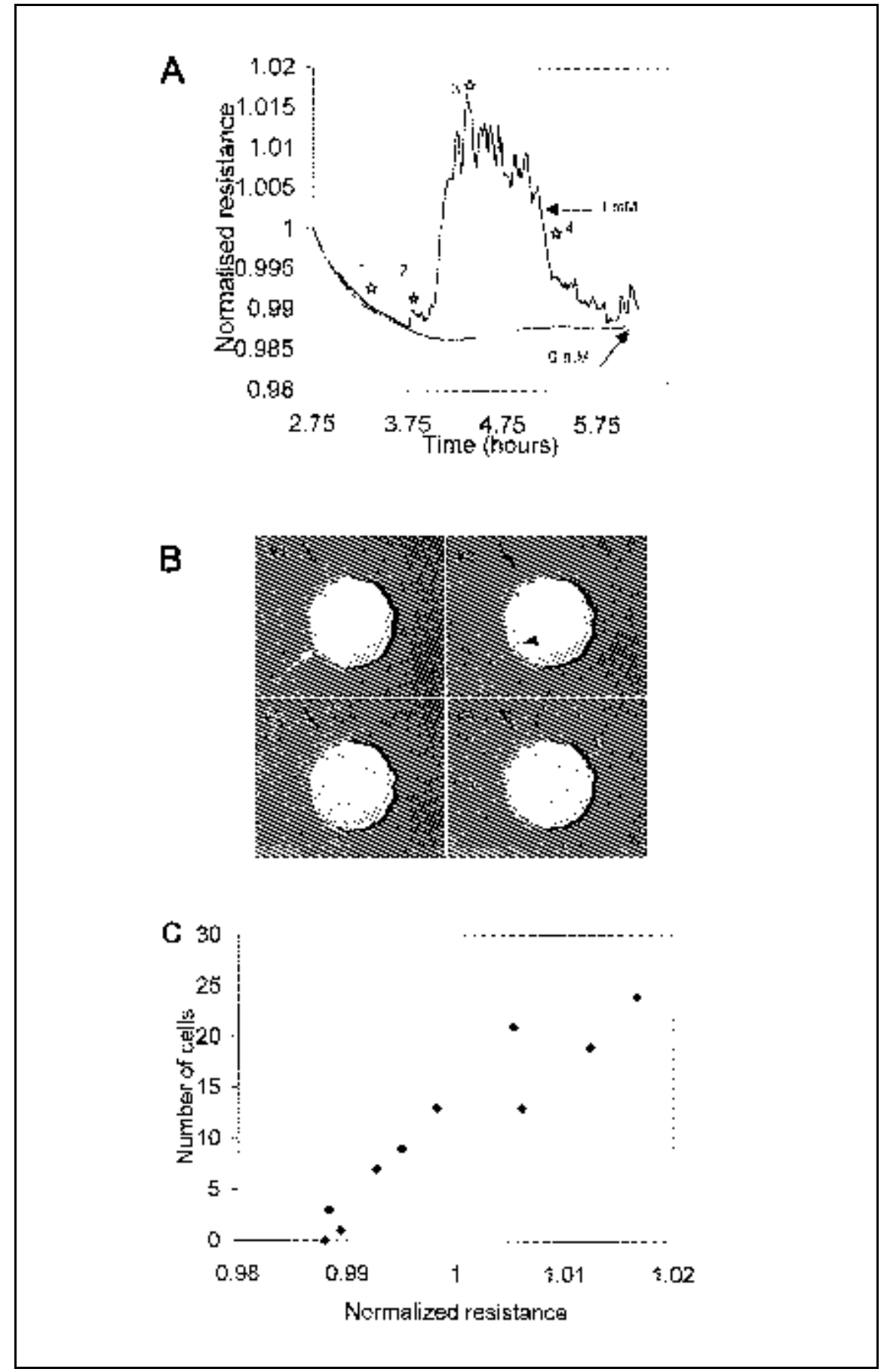

Figure 2. ECIS/taxis measurement of chemotaxis. (A) Chemotactic response to folic acid gradient. Cells were added to the cell well in the presence or absence of a folic acid gradient originating from the chemoattractant well, and the resistance is recorded over time. The data are normalized to the resistance $2.75 \mathrm{~h}$ after the addition of the cells. The resistance does not increase until the first cell reaches the gold electrode at $3.75 \mathrm{~h}$. The resistance increases dramatically as the wave of cells arrives and then decreases as the cells leave the electrode. The numbers correspond to the images in Figure 2B. These data are representative of three different experiments. (B) Digital images of the experimental chamber described in Figure 2A. Panel 1, the absence of cells on the electrode $(3.3 \mathrm{~h})$. The arrow indicates the direction of movement toward the higher concentrations of chemoattractant. Panel 2, the arrival of the first cell on the target electrode $(3.75 \mathrm{~h})$. The arrowhead indicates the arrival of the first cell on the target electrode. Panel 3 , the arrival of a wave of cells on the electrode $(4.3 \mathrm{~h})$. Panel 4 , the wave of cells leaving the electrode (5.1 h). The panels are labeled according to the time points indicated in Figure 2A. (C) Resistance values are proportional to the number of cells that are present on the electrode. The number of cells present on the target electrode at different times is plotted against the corresponding resistance values. 
tion using a Pasteur pipet.

The chemoattractant was then loaded into one well in the appropriate chambers, and the gradient was allowed to form for $1 \mathrm{~h}$. Cells were then loaded into the other well, the apparatus was attached to the ECIS instrumentation, and the measurement of resistance was initiated. A current flow of one volt a.c. at $4000 \mathrm{~Hz}$ was passed through the chamber at $60 \mathrm{~s}$ intervals. The impedance to this current flow was measured, and a resistance value was calculated according to established protocols $(4,13)$. Resistance values could be observed in real time on the ECIS/taxis system computer display. In the results presented in this paper, the data are presented as normalized resistance, which is calculated as a fraction of the initial resistance of the chamber at the start of the experiment.

\section{Imaging}

The gold electrode and overlying photoresist are thin enough to be visually transparent. For some experiments, the area of the small electrode was im aged during the collection of resistance data to establish the arrival time of cells. The ECIS/taxis plate was placed on the stage of a Leica DM IL microscope (Heidelberg, Germany) and the images were captured every minute using a Dage CCD300 video camera (Dage MTI, Michigan City, IN, USA) and a Scion CG-7 frame grabber (Scion, Frederick, MD, USA). Image capture was controlled and the images were processed using Scion Image software (a derivative of NIH Image developed by Wayne Rasband at the National Institutes of Health). In other experiments, the chambers of a plate were periodically imaged to verify the data obtained by ECIS measurements.

\section{RESULTS AND DISCUSSION}

ECIS/taxis represents a modification of the standard ECIS configuration that facilitates the automated monitoring of cell population movement over time. The standard ECIS eight-chamber slide was modified for ECIS/taxis by adding an agarose overlay that covers each chamber's surface. This enables the establishment of a chemotactic gradient between the well loaded with chemoattractant and the well in which the cells were initially loaded (Figure 1A). The cells that sense a chemoattractant

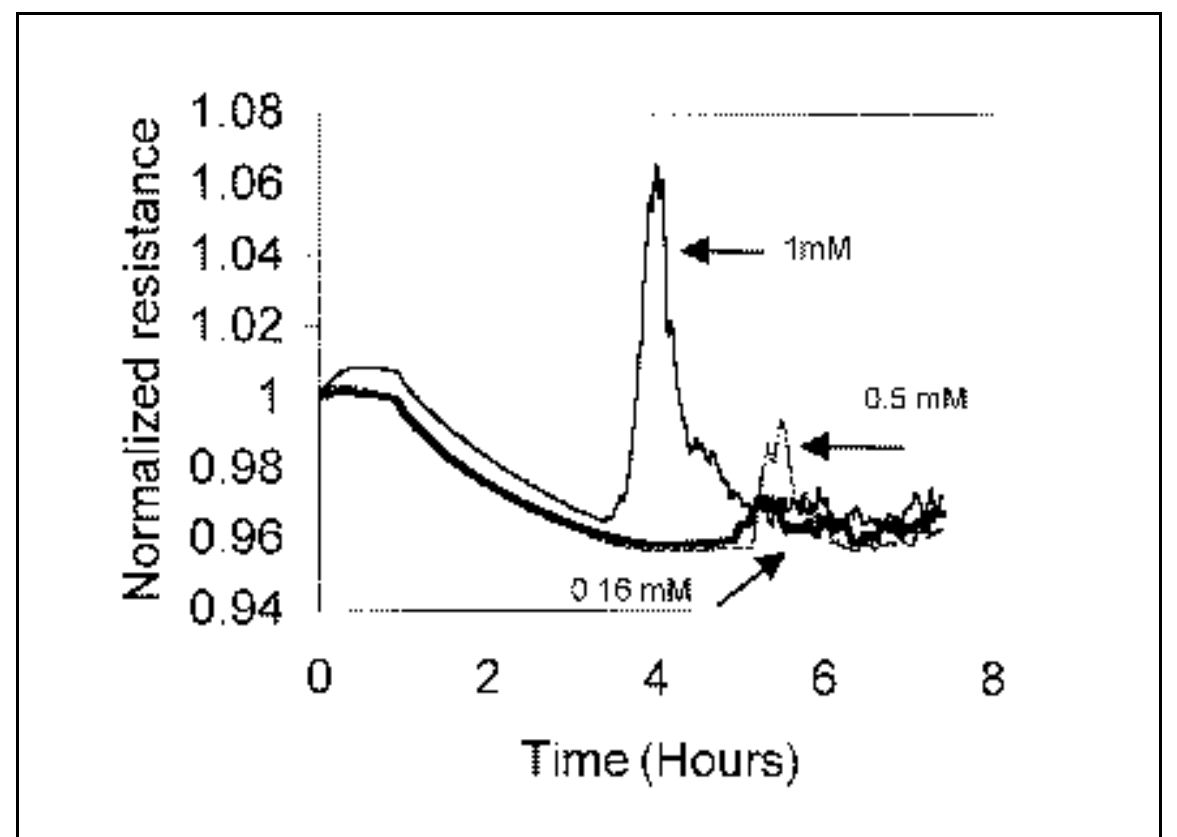

Figure 3. Chemotactic dose response to folic acid. Folic acid at $0.16,0.5$, and $1 \mathrm{mM}$ was added to the chemoattractant well $1 \mathrm{~h}$ before the addition of cells to establish the gradient. ECIS/taxis resistance values are plotted over time for the cells responding to each gradient. At suboptimal folate concentrations, fewer cells arrive at the target electrode. The data are representative of two independent experiments. 
gradient move out of the cell well under the agarose and eventually move across the target electrode located between the two wells (Figure 1B). By monitoring the changes in resistance that occur over time and the appearance of the rapid transient fluctuations in resistance, the arrival of cells at the electrode can be measured.

\section{Measurements of Cells Responding to a Simple Chemotactic Gradient}

In our initial experiments, a gradient was established by loading $1 \mathrm{mM}$ folate into the chemoattractant well of an ECIS/taxis chamber (10), and Dictyostelium cells were placed in the adjacent cell well $1 \mathrm{~h}$ later. As a control, cells were added in parallel to a well in a chamber in which no folate was added to the chemoattractant well. To visualize the arrival of the cells at the target electrode, the cells exposed to folate were continuously monitored by video microscopy. During the time before the arrival of cells at the electrode, both the control and folate chambers show a continuous, smooth decrease in resistance (Figure 2A, labeled *1). In the video images collected from this electrode, the leading cells can be seen approaching the target electrode from the lower left (Figure 2B, panel 1). The first small peak at $3.75 \mathrm{~h}$ (Figure 2A, labeled *2) coincides with the initial cell leaving the photoresist surface and spreading on the target electrode (Figure 2B, panel 2). As this first cell crawls over the electrode, the resistance remains above background and then decreases as that first cell moves off the electrode. Analysis of the time-lapse data indicates that the cell does not alter its motile behavior significantly as it changes from moving on the photoresist substrate to moving on the elemental gold electrode surface. The resistance increases again (Figure 2A, labeled *3) as a wave of cells arrives at the electrode (Figure 2B, panel 3). As the wave of cells passes, the resistance begins to gradually decrease (Figure 2B, panel 4). Throughout the period of measurement, there is a correlation between the num ber of cells on the electrode and the measured resistance values (Figure 2C).

The average speed of cell movement to the target electrode was calculated to be approximately $10 \mu \mathrm{m} / \mathrm{min}$. This speed is consistent with previous measurements of wild-type Dictyostelium chemotaxis on an agar surface (6 $\mu \mathrm{m} / \mathrm{min} \pm 1.2)(10,23)$. In the ECIS/ taxis assay, this movement occurs pri- marily on a layer of photoresist material until the cells eventually arrive at the target gold electrode surface. The cell movement is unimpeded by the $5-\mu \mathrm{m}$ step that the cells must traverse to reach the surface of the gold electrode and

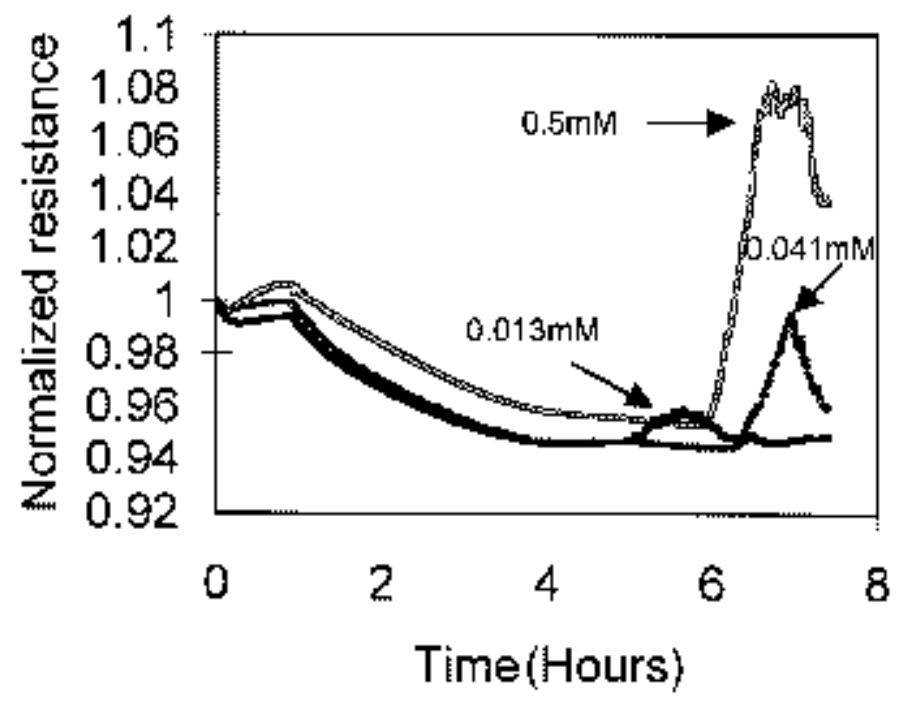

Figure 4. Cellular movement in response to a uniform folate concentration. The cells were added to the cell well in agarose premixed with folate $(0.013,0.041$, and $0.5 \mathrm{mM})$ to obtain a uniform concentration of folate throughout the chamber. Folate stimulates the motility of the cells even in the absence of a pre-established gradient. These results are representative of three independent experiments.

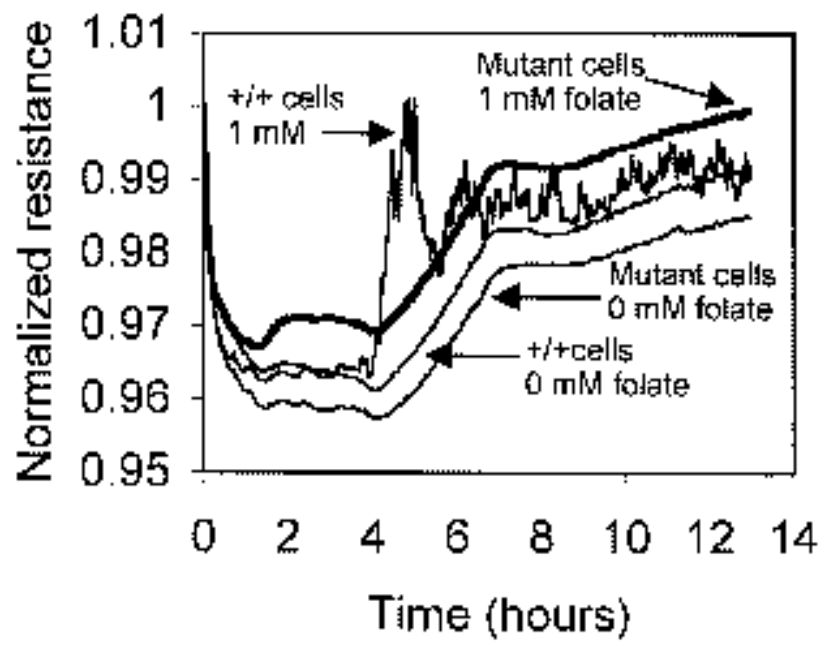

Figure 5. Characterization of the chemotactic response of mutant cells lacking myosin II heavy chain. Wild-type and myosin II null cells were added to cell wells in the presence of a 1-mM folate gradient. Only wild-type cells show a chemotactic response in the presence of folate gradient. The mutant cells never arrive at the target electrode. Similar results are obtained in three independent experiments. 
must cross again as they depart from the electrode at the other side (data not shown). It is interesting to note that the resistance does not change until the cell has actually contacted the electrode surface and is unchanged as the cell remains on the edge of the photoresist/ electrode interface. A QuickTime ${ }^{\mathrm{TM}}$ movie showing the movement of the cells across the electrode with the superim posed ECIS/taxis resistance data is available for viewing at http://www. BioTechniques.com/Movies/Nov01/ LynesECIS-taxis.html.

Before the cells arrive at the surface of the target electrode, the normalized resistance changes in a way that appears to reflect gradual changes to the electrolyte characteristics of the culture media. This change probably reflects the equilibration of the media with the external atmosphere, combined with the impact of the metabolic activity of the cultured cells on the media. Because the resistance is normalized to the initial reading within each chamber, this shift does not interfere with the ability to note the increase in resistance that attends the arrival of cells at the target electrode. Furthermore, the changes in resistance that attend changes to the culture conditions over time are not subject to the rapid transient changes in resistance that are characteristic of cellular activity on the electrode.

\section{Dictyostelium Responds to Folic Acid in a Dose-Dependent Manner}

To assess the sensitivity of this technique to the measurement of a range of chemotactic gradients, we established gradients by adding a range of folate concentrations $(0.16-1 \mathrm{mM})$ to the chemoattractant well (Figure 3). Cells exposed to control media in the absence of chemoattractant did not arrive at the target electrode during the course of the experiment; hence, no significant change to normalized resistance was observed (data not shown). In contrast, cells placed in the gradient formed by 1 $\mathrm{mM}$ folate were found to arrive at the target electrode approximately $3.5 \mathrm{~h}$ after the placement of cells in the cell well. This movement was considerably faster than the response of cells to the gradients established by either 0.5 or $0.16 \mathrm{mM}$ folate. In these instances, cell arrival at the target electrode began $5-5.25 \mathrm{~h}$ after the addition of cells to the

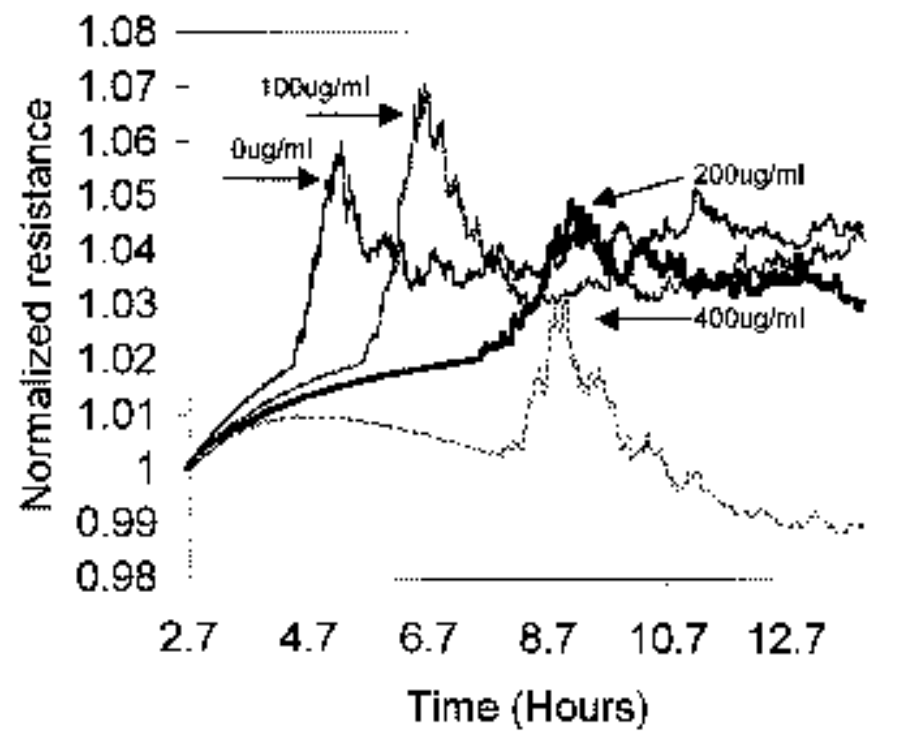

Figure 6. Delayed chemotactic response to folate after treatment with cisplatin. Wild-type cells were pretreated with cisplatin for $1 \mathrm{~h}$ at $0,100,200$, and $400 \mu \mathrm{g} / \mathrm{mL}$ before they were assessed for their response to the $1-\mathrm{mM}$ folate gradient. Increasing concentrations of cisplatin delay the arrival of cells at the electrode. The data are representative of two independent experiments. 
system. Another notable difference that distinguishes the cells responding to each level of chemoattractant is that the absolute number of cells that arrive decreases as the dose of folate used to establish the gradient decreases. This is presumably a consequence of cells that are exposed to a suboptimal concentration of folate. The advantage of ECIS/taxis over other techniques is that it allows the assessment of the whole cell population during the migratory process, as opposed to looking at the fastest cells among the population. By analyzing the entire ECIS/taxis data set, one can estimate the number of cells that have responded to the signal and the duration of time over which the cells continued to arrive at the electrode. The observation that resistance increases in a manner proportional to the number of cells on the target electrode suggests that individual resistance values can be interpreted to reveal the size of the re- sponding cell population. When testing new chemokines or inhibitors, these data will provide more information about the response of the population than a single time-point assay.

\section{ECIS/Taxis Measurements in a Uniform Concentration of Folate}

Another way to configure a chemotaxis assay is to add the chemoattractant uniformly throughout the agarose matrix. If the cells affect the chemoattractant (either by consuming it or by secreting enzymes that degrade it), then a local gradient will form (19) and cells will then move toward areas of higher chemoattractant concentration. For example, Dictyostelium secretes folate deaminase, which can destroy nearby folate and thereby create a folate gradient $(19,20)$. Cells can also respond to some agents by increasing their speed of random movement (chemokinesis), which can also result in accelerated movement away from the origin.

In the experiment shown here, folate was mixed with the agarose before it was poured into the chamber so that it was present at a uniform concentration throughout the chamber and surrounding the cell well. When the agarose contained $0.5 \mathrm{mM}$ folate, the cell arrival times at the electrode were similar to arrival times for chemotactic responses to lower concentrations of folate (Figure 4). Intriguingly, the cells arrive at the electrode as a wave rather than as a continuous stream, even though there is a large reservoir of cells that remains in the cell well. This may result from cells altering the local concentration of folate, thus limiting the movement of cells behind the initial wave. It was interesting to note that a very low concentration of folate $(0.013$ $\mathrm{mM}$ ) can accelerate the movement of a smaller number of Dictyostelium to a 
greater degree than higher concentrations. When cells are exposed to a $0.013-\mathrm{mM}$ folate concentration, they arrive at the target electrode $1-1.25 \mathrm{~h}$ earlier than they do when exposed to 0.04 or $0.5 \mathrm{mM}$ folate.

\section{ECIS/Taxis Measurements Can Identify Cell Lines that Are Unable to Produce a Chemotactic Response}

A potential experimental use for ECIS/taxis is to simplify the identification of new mutations that contribute to the chemotactic and chemokinetic processes. In a prototypical experiment, we used a myosin II mutant that has previously been shown to have a reduced ability to respond to cAMP (28) and is unable to move normally during morphogeneis (25). The response of these cells to a folate gradient has not been previously reported. The myosin II mutant cells did not arrive at the tar- get electrode at any point during the course of the experiment (Figure 5). Preliminary analysis has shown that these cells do not move far enough under agarose to reach the target electrode (Laevsky and Knecht, unpublished data). The manipulation of the gel overlay composition may allow other aspects of cellular behavior to be exam ined with this technique.

\section{Dose-Dependent Inhibition of Chemotactic Responses by Cisplatin}

Another potential use of ECIS/taxis is in the identification of pharmacological inhibitors of chemotaxis or chemokinesis. Previous work has shown that cisplatin can decrease the chemotactic responses of Dictyostelium (21). When this phenomenon was examined in the ECIS/taxis system, the arrival time of cells at the electrode was delayed in a manner proportional to the dose of cis- platin (Figure 6). While untreated cells reached the electrode after $4.4 \mathrm{~h}$, treated cells arrived at times that extended from 5.6 to $8 \mathrm{~h}$ after the start of the experiment. The drug did not have a discernable effect on cell viability at the concentrations used because it did not appear to affect the number of cells that eventually arrived at the electrode. Cisplatin may inhibit cell movement through the inhibition of the association of actin with the cortex (21) and/or via interactions with the signal transduction cascade $(12,21)$. This result illustrates the potential of ECIS/taxis for high-throughput screening of potential agonists and antagonists of chemotactic behavior.

\section{CONCLUSION}

The analysis of cell movement in the presence of chemotactic and chemokinetic stimuli is relevant to many differ- 
ent lines of basic and applied research. One of the primary uses of this technology will be for the study of normal immune processes (16) and disease processes including chronic inflammation, autoimmune disease (22), and cancer. Because ECIS/taxis can rely on the computerized assessment of experimental results according to an objective algorithm and is also amenable to robotic setup and data capture, it will likely prove to be valuable in many pharmaceutical and biotechnology applications, including the evaluation of anti-inflammatory drugs and the clinical evaluation of patient immune function. It is also possible that adaptations of the techniques described in this report can be used to assess the movement of neoplastic cells and drugs that alter that form of cellular movement $(8,14,24)$. Finally, scaling up the ECIS/taxis system to larger numbers of chambers may also allow highthroughput screening of mutant cells that have alterations in their chemotactic response, enabling the rapid identification of genes involved in the regulation of cellular movement.

\section{ACKNOWLEDGMENTS}

This work was supported by National Institutes of Health grant no. AI46790 to M.A.L. and grant no. GM40599 to D.A.K.

\section{REFERENCES}

1.Boyden, S. 1962. The chemotactic effect of mixtures of antibody and antigen on polymorphonuclear leukocytes. J. Exp. Med. 152:453466.

2.Devreotes, P.N. 1983. Cyclic nucleotides and cell-cell communication in Dictystelium discoideum Adv. Cyclic Nucleotide Res. 15:453-496.

3.Devreotes, P.N. and S.H. Zigmond. 1988. Chemotaxis in eukaryotic cells: a focus on leukocytes and Dictyostelium. Annu. Rev. Cell Biol. 4:649-686.

4.Ghosh, P.M., C.R. Keese, and I. Giaever. 1994. Morphological response of mammalian cells to ac fields. Bioelectrochem. Bioenerg. $33: 121-133$.

5.Giaever, I. and C.R. Keese. 1992. Toxic? Cells can tell. Chemtech. 22:116-125.

6.Keese, C.R. and I. Giaever. 1990. A whole cell biosensor based on cell substrate interactions. IEEE Eng. Med. Biol. Mag. 12:500501.
7.Konijn, T.M. and P.J. Van Haastert. 1987. Measurement of chemotaxis in Dictyostelium. Methods Cell Biol. 28:283-298.

8.Koshiba, T., R. Hosotani, Y. Miyamoto, J. Ida, S. Tsuji, S. Nakajima, M. Kawaguchi, H. Kobayashi et al. 2000. Expression of stromal cell-derived factor 1 and CXCR4 ligand receptor system in pancreatic cancer: a possible role for tumor progression. Clin. Cancer Res. 6:3530-3535.

9.Kowolenko, M., C.R. Keese, D.A. Law rence, and I. Giaever. 1990. Measurement of macrophage adherence and spreading with weak electric fields. J. Immunol. Methods 127:71-77.

10.Laevsky, G. and D.A. Knecht. 2001. UnderAgarose folate chemotaxis of Dictyostelium amoebae in permissive and mechanically inhibited conditions. BioTechniques 31:11401149.

11.Lauffenburger, D.A., R.T. Tranquillo, and H.S. Zigmond. 1988. Concentration gradients of chemotactic factors in chemotaxis assays. Methods Enzymol. 162:85-101.

12.Li, G., H. Alexander, N. Schneider, and S. Alexander. 2000. Molecular basis for resistance to the anticancer drug cisplatin in Dictyostelium. Microbiology 146:2219-2227.

13.Lo, C.M., C.R. Keese, and I. Giaever. 1993. Monitoring motion of confluent cells in tissue culture. Exp. Cell Res. 204:102-109.

14.Morita, M., S. Tsuruta, K.J. Mori, M. Mayumi, and H. Mikawa. 1990. Ketotifen inhibits PAF-induced actin polymerization in a human eosinophilic leukaemia cell line, EoL-1. Eur. Respir. J. 3:1173-1178.

15.Morrison, A. and A. Harwood. 1992. A sim ple method of generating axenic derivatives of Dictyostelium strains. Exp. Cell Res. 199:383-386.

16.Moser, B. and P. Loetscher. 2001. Lymphocyte traffic control by chemokines. Nat. Im munol. 2:123-128.

17.Nelson, R.D., P.G. Quie, and R.L. Simmons. 1975. Chemotaxis under agarose: a new and simple method for measuring chemotaxis and spontaneous migration of human polymorphonuclear leukocytes and monocytes. J. Im munol. 115:1650-1656.

18.Newton-Nash, D.K., P. Tonellato, M. Swiersz, and P. Abramoff. 1990. Assessment of chemokinetic behavior of inflammatory lung macrophages in a linear under-agarose assay. J. Leukoc. Biol. 48:297-305.

19.Pan, P., E.M. Hall, and J.T. Bonner. 1972. Folic acid as second chemotactic substance in the cellular slime molds. Nat. New Biol. 237:181-182.

20.Pan, P. and B. Wurster. 1978. Inactivation of the chemoattractant folic acid by cellular slime molds and identification of the reaction product. J. Bacteriol. 136:955-959.

21.Reddy, T.B. and S. Chatterjee. 1999. Cisplatin inhibits folic acid chemotaxis and phagocytotic functions in Dictyostelium discoideum Cell Biol. Int. 23:227-233.

22.Sasagawa, S., Y. Satow, K. Suzuki, and T. Hosokawa. 1992. Chemotactic activities of peripheral blood polymorphonuclear leukocytes and peritoneal exudate polymorphonuclear leukocytes in MRL mice. Immunopharmacol. Immunotoxicol. 14:625-635.
23.Segall, J.E., P.R. Fisher, and G. Gerisch. 1987. Selection of chemotaxis mutants of Dictyostelium discoideum J. Cell Biol. 104:151161.

24.Sezzi, M.L., G. De Luca, M. Materazzi, and L. Bellelli. 1985. Effects of a calcium-antagonist (flunarizine) on cancer cell movement and phagocytosis. Anticancer Res. 5:265-271.

25.Shelden, E. and D.A. Knecht. 1995. Mutants lacking myosin II cannot resist forces generated during multicellular morphogenesis. J. Cell Sci. 108:1105-1115.

26.Sussman, R. and M. Sussman. 1967. Cultivation of Dictyostelium discoideumin axenic medium. Biochem. Biophys. Res. Commun. 29:53-55.

27.Wegener, J., C.R. Keese, and I. Giaever. 2000. Electric cell-substrate impedance sensing (ECIS) as a noninvasive means to monitor the kinetics of cell spreading to artificial surfaces. Exp. Cell Res. 259:158-166.

28. Wessels, D., D.R. Soll, D. Knecht, W.F. Loomis, A. De Lozanne, and J. Spudich. 1988. Cell motility and chemotaxis in Dictyostelium amebae lacking myosin heavy chain. Dev. Biol. 128:164-177.

29.Zicha, D., G.A. Dunn, and A.F. Brown. 1991. A new direct-viewing chemotaxis chamber. J. Cell Sci. 99:769-775.

30.Zigmond, S.H. 1988. Orientation chamber in chemotaxis. Methods Enzymol. 162:65-72.

Received 31 January 2001; accepted 15 May 2001.

Address correspondence to:

Dr. Michael A. Lynes

U-125, University of Connecticut

Department of Molecular and Cell Biology

75 North Eagleville Road

Storrs, CT 06269-3125, USA

e-mail: lynes@uconnvm.uconn.edu

For reprints of this or any other article, contact Reprints@BioTechniques.com 